\title{
Scale-Invariant Fully Homomorphic Encryption over the Integers
}

\author{
Jean-Sébastien Coron $^{1}$, Tancrède Lepoint ${ }^{1,2,3}$, and Mehdi Tibouchi ${ }^{4}$ \\ 1 University of Luxembourg, Luxembourg \\ jean-sebastien.coron@uni.lu \\ 2 École Normale Supérieure, France \\ 3 CryptoExperts, France \\ tancrede.lepoint@cryptoexperts.com \\ 4 NTT Secure Platform Laboratories, Japan \\ tibouchi.mehdi@lab.ntt.co.jp
}

\begin{abstract}
At Crypto 2012, Brakerski constructed a scale-invariant fully homomorphic encryption scheme based on the LWE problem, in which the same modulus is used throughout the evaluation process, instead of a ladder of moduli when doing "modulus switching". In this paper we describe a variant of the van Dijk et al. FHE scheme over the integers with the same scale-invariant property. Our scheme has a single secret modulus whose size is linear in the multiplicative depth of the circuit to be homomorphically evaluated, instead of exponential; we therefore construct a leveled fully homomorphic encryption scheme. This scheme can be transformed into a pure fully homomorphic encryption scheme using bootstrapping, and its security is still based on the ApproximateGCD problem.

We also describe an implementation of the homomorphic evaluation of the full AES encryption circuit, and obtain significantly improved performance compared to previous implementations: about 23 seconds (resp. 3 minutes) per AES block at the 72-bit (resp. 80-bit) security level on a mid-range workstation.

Finally, we prove the equivalence between the (error-free) decisional Approximate-GCD problem introduced by Cheon et al. (Eurocrypt 2013) and the classical computational Approximate-GCD problem. This equivalence allows to get rid of the additional noise in all the integer-based FHE schemes described so far, and therefore to simplify their security proof.
\end{abstract}

\section{Introduction}

Fully Homomorphic Encryption. In 2009, Gentry constructed the first fully homomorphic encryption scheme (FHE), i.e. a scheme allowing a worker to evaluate any circuit on plaintext values while manipulating only ciphertexts. The first generation of FHE schemes Gen09, DGHV10, SV10, GH11, BV11a, BV11b and [CMNT11, CNT12, $\left.\mathrm{CCK}^{+} 13\right]$ followed Gentry's blueprint to achieve a fully homomorphic scheme. 
The first step of Gentry's blueprint is to construct a somewhat homomorphic encryption scheme (SWHE) capable of evaluating "low degree" polynomials homomorphically. Inherent to this construction is the property that ciphertexts are "noisy", and noises grow slightly with homomorphic additions and substantially with homomorphic multiplications. Thus ciphertexts need to be refreshed to maintain a low noise level and allow subsequent homomorphic operations. To obtain a FHE scheme, Gentry's key-idea, referred to as bootstrapping, states that a SWHE capable of evaluating its own decryption procedure (and an additional multiplication) can be transformed into a FHE scheme. Bootstrapping consists in evaluating the decryption circuit of the SWHE scheme using the decryption key bits in encrypted form, thus resulting in a different encryption of the same plaintext but with reduced noise. In practice, the scheme parameters are generally determined so that the refreshed ciphertexts can handle one additional homomorphic multiplication GH11, CMNT11, CNT12, $\mathrm{CCK}^{+} 13$. Unfortunately, the downside of these settings is that one needs to call the (very costly) bootstrapping procedure after each homomorphic multiplication.

Modulus Switching and Scale Invariance. To avoid bootstrapping a new noise management technique, called modulus switching, was introduced by Brakerski, Gentry and Vaikuntanathan BGV12. The authors obtained a leveled FHE scheme: i.e. a scheme in which the noise grows linearly with the multiplicative depth instead of exponentially as in somewhat homomorphic encryption. Therefore any circuit with polynomial depth can be evaluated. The technique consists in scaling down the noise by converting a ciphertext modulo $q$ into a ciphertext modulo a smaller $q^{\prime}$; the noise being reduced by roughly a factor $q / q^{\prime}$. By carefully calibrating the ladder of moduli, the noise growth can then be made linear with the number of homomorphic multiplications. The technique was also adapted to the DGHV fully homomorphic encryption scheme over the integers DGHV10 in CNT12. Unfortunately for a circuit with $L$ layers of multiplication, the technique requires to store the equivalent of $L$ public-keys, yielding a huge storage requirement.

At Crypto 2012, Brakerski introduced a new tensor product technique for LWE-based leveled FHE Bra12] so that the same modulus is used throughout the evaluation process instead of a layer of moduli; the noise growth is still linear in the number of homomorphic multiplications. This was achieved by considering ciphertexts such that $\langle\boldsymbol{c}, \boldsymbol{s}\rangle=\lfloor q / 2\rfloor \cdot m+e \bmod q$, instead of $\langle\boldsymbol{c}, \boldsymbol{s}\rangle=$ $m+2 e \bmod q$, as in Regev's initial scheme Reg05.

Implementations of FHE Schemes. Independently at Crypto 2012, Gentry et al. benchmarked a LWE-based scheme by homomorphically evaluating an AES circuit GHS12b], yielding to the first "real-world" circuit homomorphically evaluated by a FHE scheme. This implementation used the modulus switching technique of [BGV12] and additionally a batching technique [SV11, BGV12, GHS12a that allows one to encrypt vectors of plaintexts in a single ciphertext, and to perform any permutation on the underlying plaintext vector while manipulating only the ciphertext. They obtained a timing of about 5 minutes per AES block 
homomorphically encrypted. Similar results were later obtained for the integerbased DGHV scheme [DGHV10, extending the batching technique and homomorphically evaluating AES on a desktop computer in about 12 minutes per block for 72 bits of security $\mathrm{CCK}^{+} 13$, CLT13.

Our Contributions. In this paper, we describe a variant of the DGHV scheme over the integers with the same scale-invariant property as in Bra12; i.e. our scheme does not use modulus switching and the noise grows linearly with the multiplicative depth. We obtain a DGHV variant with a single secret modulus $p$ whose size is linear in the multiplicative depth (instead of exponential). Our technique is as follows.

In the original DGHV scheme, a ciphertext $c$ of the bit message $m \in\{0,1\}$ has the form

$$
c=m+2 r+q \cdot p,
$$

where $p$ is the secret key, $q$ is a large random integer, and $r$ is a small random integer (noise). The bit message is recovered by computing $m=(c \bmod p) \bmod 2$. Adding and multiplying ciphertexts over $\mathbb{Z}$ respectively adds and multiplies the plaintexts modulo 2 while keeping them hidden. Unfortunately, the noise grows exponentially with the number of homomorphic multiplications: if two ciphertexts $c_{1}, c_{2}$ have $\rho$-bit noise, the noise of $c_{3}=c_{1} \cdot c_{2}$ has $\approx 2 \rho$ bits. Therefore to evaluate a circuit with $L$ sequential layers of multiplications without bootstrapping, the bit-size $\eta$ of the modulus $p$ must satisfy $\eta>2^{L} \rho$.

In our new scheme, similar to Bra12, instead of encrypting the bit $m \in\{0,1\}$ in the LSB of $[c \bmod p]$, we encrypt it in the MSB of $[c \bmod p]$; additionally we work modulo $p^{2}$ instead of modulo $p$. More precisely, the message $m$ is now encrypted as

$$
c=r+\left(m+2 r^{*}\right) \cdot \frac{p-1}{2}+q \cdot p^{2},
$$

where the ciphertext now contains two noises $r$ and $r^{*}$. We decrypt $c$ by computing $m=(2 c \bmod p) \bmod 2$. Clearly adding two ciphertexts over $\mathbb{Z}$ still adds the underlying bit messages $m$ modulo 2 . However, multiplication of two ciphertexts moves the bit message $m$ from the MSB of $[c \bmod p]$ to the MSB of $\left[c \bmod p^{2}\right]$. Namely, a ciphertext $c$ obtained as the multiplication of ciphertexts $c_{1}$ and $c_{2}$ for the respective bit messages $m_{1}$ and $m_{2}$ will have the form

$$
c=2 \cdot c_{1} \cdot c_{2}=r+\left(m_{1} \cdot m_{2}\right) \cdot \frac{p^{2}-1}{2}+q \cdot p^{2},
$$

where $r>p$ but still $r \ll p^{2}$. We then describe a procedure Convert that allows to publicly convert the result of a multiplication (i.e. a ciphertext as in Equation (2) ) into a ciphertext reusable in subsequent homomorphic operations (i.e. a ciphertext as in Equation (10), either keeping the same secret $p$ (which requires, as usual, a circular security assumption) or using a different fresh $p$ at each level (which requires a larger secret key). The bit length of the noise in the new ciphertext grows only by a constant additive factor with respect to the noise in $c_{1}$ and $c_{2}$ (see Figure 1 for an illustration). Therefore, our scheme 


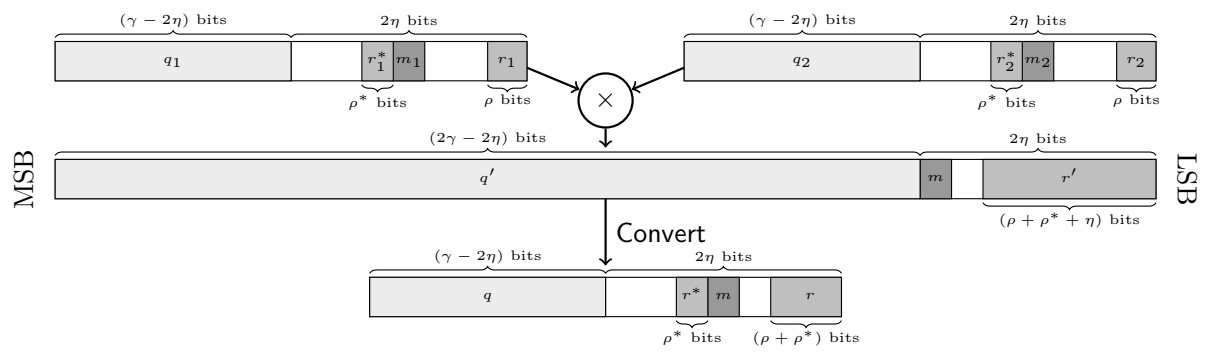

Fig. 1. Conversion of a ciphertext after a homomorphic multiplication

is a variant of the DGHV scheme that is a leveled fully homomorphic encryption scheme. It can be turned into a pure FHE scheme using bootstrapping (cf. DGHV10, CMNT11, CNT12, $\left.\mathrm{CCK}^{+} 13\right]$ ). We also show that our scheme is semantically secure, under the Approximate-GCD assumption.

We also adapt our scale-invariant technique to the batch setting in $\mathrm{CCK}^{+} 13$ and homomorphically evaluate an AES encryption as in GHS12b, $\mathrm{CCK}^{+13}$. Our scheme offers competitive performances as it can evaluate the full AES circuit in about 23 seconds (resp. 3 minutes) per AES block at the 72-bit (resp. 80-bit) security level on a mid-range workstation, that is one order of magnitude faster than $\left.\mathrm{CCK}^{+} 13\right]$.

Finally, we prove the equivalence between the (error-free) computational Approximate-GCD problem DGHV10 and the (error-free) decisional Approximate-GCD problem introduced in $\left[\mathrm{CCK}^{+} 13\right.$, KLYC13. From this equivalence, the additional noise added during encryption to drawn the noises coming from the public key elements is no longer required. This yields automatic improvements in the parameters of all the fully homomorphic encryption schemes over the integers.

\section{The Somewhat Homomorphic DGHV Scheme}

In this section we first recall the somewhat homomorphic encryption scheme over the integers of van Dijk, Gentry, Halevi and Vaikuntanathan (DGHV) in DGHV10]. We denote by $\lambda$ the security parameter, $\tau$ the number of elements in the public key, $\gamma$ their bit-length, $\eta$ the bit-length of the secret key $p$ and $\rho$ (resp. $\rho^{\prime}$ ) the bit-length of the noise in the public key (resp. in a fresh ciphertext).

For a real number $x$, we denote by $\lceil x\rceil,\lfloor x\rfloor$ and $\lceil x\rfloor$ the upper, lower or nearest integer part of $x$. For integers $z, p$ we denote the reduction of $z$ modulo $p$ by $(z \bmod p)$ or $[z]_{p}$ with $-p / 2<[z]_{p} \leqslant p / 2$. For a specific $\eta$-bit odd integer $p$, we use the following distribution over $\gamma$-bit integers:

$$
\mathcal{D}_{\gamma, \rho}(p)=\left\{\begin{array}{c}
\text { Choose } q \leftarrow \mathbb{Z} \cap\left[0,2^{\gamma} / p\right), r \leftarrow \mathbb{Z} \cap\left(-2^{\rho}, 2^{\rho}\right): \\
\text { Output } x=q \cdot p+r
\end{array}\right\} .
$$


DGHV. KeyGen $\left(1^{\lambda}\right)$. Generate an $\eta$-bit random prime integer $p$. For $0 \leqslant i \leqslant \tau$, sample $x_{i} \leftarrow \mathcal{D}_{\gamma, \rho}(p)$. Relabel the $x_{i}$ 's so that $x_{0}$ is the largest. Restart unless $x_{0}$ is odd and $\left[x_{0}\right]_{p}$ is even. Let $\mathrm{pk}=\left(x_{0}, x_{1}, \ldots x_{\tau}\right)$ and $\mathrm{sk}=p$.

DGHV. Encrypt(pk, $m \in\{0,1\})$. Choose a random subset $S \subseteq\{1,2, \ldots, \tau\}$ and a random integer $r$ in $\left(-2^{\rho^{\prime}}, 2^{\rho^{\prime}}\right)$, and output the ciphertext:

$$
c=\left[m+2 r+2 \sum_{i \in S} x_{i}\right]_{x_{0}} .
$$

DGHV. Evaluate(pk, $\left.C, c_{1}, \ldots, c_{t}\right)$. Given the circuit $C$ with $t$ input bits and $t$ ciphertexts $c_{i}$, apply the addition and multiplication gates of $C$ to the ciphertexts, performing all the additions and multiplications over the integers, and return the resulting integer.

DGHV. Decrypt $($ sk, $c)$. Output $m \leftarrow(c \bmod p) \bmod 2$.

This completes the description of the scheme. The scheme is clearly somewhat homomorphic, i.e. a limited number of homomorphic operations can be performed on ciphertexts. More precisely given two ciphertexts $c=q \cdot p+2 r+m$ and $c^{\prime}=q^{\prime} \cdot p+2 r^{\prime}+m^{\prime}$ where $r$ and $r^{\prime}$ are $\rho^{\prime}$-bit integers, the ciphertext $c+c^{\prime}$ is an encryption of $m+m^{\prime} \bmod 2$ with a $\left(\rho^{\prime}+1\right)$-bit noise and the ciphertext $c \cdot c^{\prime}$ is an encryption of $m \cdot m^{\prime}$ with noise bit-length $\simeq 2 \rho^{\prime}$. Therefore the scheme allows roughly $\eta / \rho^{\prime}$ successive multiplications on ciphertexts (since the noise must remain smaller than $p$ for correct decryption).

As shown in DGHV10] the scheme is semantically secure under the Approximate-GCD assumption.

Definition 1 (Approximate GCD). The $(\rho, \eta, \gamma)$-Approximate-GCD problem consists, given a random $\eta$-bit odd integer $p$ and given polynomially many samples from $\mathcal{D}_{\gamma, \rho}(p)$, in outputting $p$.

\section{Scale-Invariant DGHV Scheme}

In this section we describe our variant of the DGHV scheme with the scaleinvariant property. We first explain the two main ideas of our scheme, namely 1) moving the plaintext bit from the LSB to the MSB of $[c \bmod p]$ and working modulo $p^{2}$, and 2 ) converting the result of a ciphertext multiplication back to a ciphertext usable in subsequent homomorphic operations. We then provide the full description of our scheme.

\subsection{Ciphertexts and Homomorphic Operations}

As explained in introduction, instead of encrypting the plaintext $m \in\{0,1\}$ in the LSB of $[c \bmod p], m$ is now encrypted in the MSB of $[c \bmod p]$ as

$$
c=r+\left(m+2 r^{*}\right) \cdot \frac{p-1}{2}+q \cdot p^{2}
$$


where the ciphertext has now two noises $r$ and $r^{*}$ of respective bit-length $\rho$ and $\rho^{*}$. We call such ciphertext a Type-I ciphertext and we say that $c$ has noise length $\left(\rho, \rho^{*}\right)$. To decrypt $c$, one computes $(2 c \bmod p) \bmod 2=m$.

Homomorphic additions are performed as additions over $\mathbb{Z}$ : namely given two Type-I ciphertexts $c_{1}$ and $c_{2}$ of noise $\left(\rho, \rho^{*}\right)$ :

$$
\begin{aligned}
& c_{1}=r_{1}+\left(m_{1}+2 r_{1}^{*}\right) \cdot(p-1) / 2+q_{1} \cdot p^{2} \\
& c_{2}=r_{2}+\left(m_{2}+2 r_{2}^{*}\right) \cdot(p-1) / 2+q_{2} \cdot p^{2}
\end{aligned}
$$

we get

$$
c_{1}+c_{2}=r_{3}+\left(m_{1}+m_{2}+2 r_{3}^{*}\right) \cdot \frac{p-1}{2}+q_{3} \cdot p^{2},
$$

for some integers $r_{3}, r_{3}^{*}$ and $q_{3}$, with $\log _{2}\left|r_{3}\right| \leqslant \rho+1$ and $\log _{2}\left|r_{3}^{*}\right| \leqslant \rho^{*}+1$.

Next, to homomorphically multiply the ciphertexts $c_{1}$ and $c_{2}$, one computes $c_{3}=2 \cdot c_{1} \cdot c_{2}$ over $\mathbb{Z}$. This gives

$$
\begin{aligned}
c_{3}=2 \cdot c_{1} \cdot c_{2}= & 2 r_{1} r_{2}+\left(r_{1}\left(m_{2}+2 r_{2}^{*}\right)+r_{2}\left(m_{1}+2 r_{1}^{*}\right)\right) \cdot(p-1)+ \\
& \left(m_{1}+2 r_{1}^{*}\right) \cdot\left(m_{2}+2 r_{2}^{*}\right) \cdot \frac{(p-1)^{2}}{2}+q_{3}^{\prime} \cdot p^{2} \\
= & r_{3}^{\prime}+\left(m_{1}+2 r_{1}^{*}\right) \cdot\left(m_{2}+2 r_{2}^{*}\right) \cdot \frac{(p-1)^{2}}{2}+q_{3}^{\prime} \cdot p^{2}
\end{aligned}
$$

for some integers $q_{3}^{\prime}$ and $r_{3}^{\prime}$, with $\log _{2}\left|r_{3}^{\prime}\right| \leqslant \eta+\rho+\rho^{*}+3$, where $\eta$ is the bit-size of $p$. We use $\eta \gg \rho, \rho^{*}$. Then, there exist integers $r_{3}$ and $q_{3}$ such that

$$
c_{3}=r_{3}+m_{3} \cdot \frac{p^{2}-1}{2}+q_{3} \cdot p^{2},
$$

where $m_{3}=m_{1} \cdot m_{2}$. We call an integer $c$ verifying Equation (2) a Type-II ciphertext. The bit-length of noise $r_{3}$ satisfies $\log _{2}\left|r_{3}\right| \leqslant \eta+\rho+\rho^{*}+4$, assuming $\rho^{*}<\rho$. We refer to Figure 1 for a graphical representation of the homomorphic multiplication.

\subsection{Conversion from Type-II Ciphertext to Type-I Ciphertext}

We show that we can efficiently convert a Type-II ciphertext back to a TypeI ciphertext, using only the public-key. Our procedure Convert uses essentially the same technique as the modulus switching technique for DGHV in CNT12. Namely modulus switching in CNT12 enables to convert a classical DGHV ciphertext modulo a prime $p$ into a new ciphertext modulo a prime $p^{\prime}$, with noise scaled by a factor $p^{\prime} / p$. Similarly, our Convert procedure converts a Type-II ciphertext modulo $p^{2}$ back to a ciphertext where the noise is modulo $p$ (therefore the noise is scaled by a factor $\left.p / p^{2}=1 / p\right)$, but still somehow encrypted modulo $p^{2}$.

More precisely, we start from a Type-II ciphertext:

$$
c=r+\frac{p^{2}-1}{2} \cdot m+q \cdot p^{2}
$$


where $|r| \leqslant 2^{\rho^{\prime}}$. Let $\kappa$ be such that $|c|<2^{\kappa}$. Let $\boldsymbol{z}$ be a vector of $\Theta$ rational numbers in $\left[0,2^{\eta}\right)$ with $\kappa$ bits of precision after the binary point, and let $s$ be a vector of $\Theta$ bits such that

$$
\frac{2^{\eta}}{p^{2}}=\langle\boldsymbol{s}, \boldsymbol{z}\rangle+\varepsilon \quad \bmod 2^{\eta},
$$

where $|\varepsilon| \leqslant 2^{-\kappa}$. Here $\Theta$ is a parameter to be chosen later for security. We use the same BitDecomp and PowersofTwo procedures as in BGV12.

- BitDecomp $\eta_{\eta}(\boldsymbol{v}):$ For $\boldsymbol{v} \in \mathbb{Z}^{n}$, let $\boldsymbol{v}_{i} \in\{0,1\}^{n}$ be such that $\boldsymbol{v} \bmod 2^{\eta}=$ $\sum_{i=0}^{\eta-1} \boldsymbol{v}_{i} \cdot 2^{i}$. Output the vector

$$
\left(\boldsymbol{v}_{0}, \ldots, \boldsymbol{v}_{\eta-1}\right) \in\{0,1\}^{n \cdot \eta}
$$

- PowersofTwo $w_{\eta}(\boldsymbol{w}):$ For $\boldsymbol{w} \in \mathbb{Z}^{n}$, output the vector

$$
\left(\boldsymbol{w}, 2 \cdot \boldsymbol{w}, \ldots, 2^{\eta-1} \cdot \boldsymbol{w}\right) \in \mathbb{Z}^{n \cdot \eta}
$$

Given the vector $s$ from (5), we let $\boldsymbol{s}^{\prime}=\operatorname{PowersofT}_{\eta}(s)$, and let

$$
\boldsymbol{\sigma}=\boldsymbol{q} \cdot p^{2}+\boldsymbol{r}+\left\lfloor\boldsymbol{s}^{\prime} \cdot \frac{p}{2^{\eta+1}}\right\rceil
$$

be an "encryption" of the vector $\boldsymbol{s}^{\prime}$, where $\boldsymbol{q} \leftarrow\left(\mathbb{Z} \cap\left[0,2^{\gamma} / p^{2}\right)\right)^{\eta \cdot \Theta}$ and $\boldsymbol{r} \leftarrow$ $\left(\mathbb{Z} \cap\left(-2^{\rho}, 2^{\rho}\right)\right)^{\eta \cdot \Theta}$. We can now define the Convert algorithm:

Convert $(\boldsymbol{z}, \boldsymbol{\sigma}, c)$. First compute $\boldsymbol{c}=\left(\left\lfloor c \cdot z_{i}\right\rceil \bmod 2^{\eta}\right)_{1 \leqslant i \leqslant \Theta}$ and its decomposition $\boldsymbol{c}^{\prime}=\operatorname{BitDecomp}_{\eta}(\boldsymbol{c})$. Finally, output

$$
c^{\prime} \leftarrow 2\left\langle\boldsymbol{\sigma}, \boldsymbol{c}^{\prime}\right\rangle
$$

The following Lemma shows that our procedure Convert enables one to transform a Type-II ciphertext back to a Type-I ciphertext. We provide the proof in the full version of the paper CLT14.

Lemma 1. Let $\rho^{\prime}$ be such that $\rho^{\prime} \geqslant \eta+\rho+\log _{2}(\eta \Theta)$. The procedure Convert above converts a Type-II ciphertext with noise size $\rho^{\prime}$ into a Type-I ciphertext with noise $\left(\rho^{\prime}-\eta+5, \log _{2} \Theta\right)$.

Assume that initially the two ciphertexts $c_{1}, c_{2}$ are Type-I ciphertexts with noise $\left(\rho_{1}, \log _{2} \Theta\right)$. After computing $c_{3}=2 \cdot c_{1} \cdot c_{2}$ which has noise size at most $\rho^{\prime}=\eta+\rho_{1}+\log _{2} \Theta+4$ (see previous section) one can convert $c_{3}$ back into a Type-I ciphertext with noise $\left(\rho_{3}, \rho_{3}^{*}\right)$ with $\rho_{3}=\rho_{1}+\log _{2} \Theta+9$ and $\rho_{3}^{*}=\log _{2} \Theta$, from Lemma 1. Therefore the noise length in bits has only grown by an additive factor $\log _{2} \Theta+9$. Therefore the ciphertext noise grows only linearly with the number of homomorphic multiplications. 


\subsection{Description of the Public-Key Leveled Fully Homomorphic Scheme}

We are now ready to describe our scale-invariant version of the DGHV encryption scheme. For a specific $\eta$-bit odd integer $p$ and an integer $q_{0}$ in $\left[0,2^{\gamma} / p^{2}\right)$, we define the set:

$$
\mathcal{D}_{p, q_{0}}^{\rho}=\left\{q \cdot p^{2}+r: q \in \mathbb{Z} \cap\left[0, q_{0}\right), r \in \mathbb{Z} \cap\left(-2^{\rho}, 2^{\rho}\right)\right\} .
$$

SIDGHV. KeyGen $\left(1^{\lambda}\right)$. Generate an odd $\eta$-bit integer $p$ and a $\gamma$-bit integer $x_{0}=$ $q_{0} \cdot p^{2}+r_{0}$ with $r_{0} \leftarrow\left(-2^{\rho}, 2^{\rho}\right) \cap \mathbb{Z}$ and $q_{0} \leftarrow\left[0,2^{\gamma} / p^{2}\right) \cap \mathbb{Z}$. Let $x_{i} \leftarrow \mathcal{D}_{p, q_{0}}^{\rho}$ for $1 \leqslant i \leqslant \tau$. Let also $y^{\prime} \leftarrow \mathcal{D}_{p, q_{0}}^{\rho}$ and $y=y^{\prime}+(p-1) / 2$.

Let $\boldsymbol{z}$ be a vector of $\Theta$ numbers with $\kappa=2 \gamma+2$ bits of precision after the binary point, and let $s$ be a vector of $\Theta$ bits such that

$$
\frac{2^{\eta}}{p^{2}}=\langle\boldsymbol{s}, \boldsymbol{z}\rangle+\varepsilon \bmod 2^{\eta},
$$

with $|\varepsilon| \leqslant 2^{-\kappa}$. Now, define

$$
\boldsymbol{\sigma}=\boldsymbol{q} \cdot p^{2}+\boldsymbol{r}+\left\lfloor\text { PowersofT }_{\mathrm{wo}_{\eta}}(\boldsymbol{s}) \cdot \frac{p}{2^{\eta+1}}\right\rceil,
$$

where the components of $\boldsymbol{q}$ (resp. $\boldsymbol{r}$ ) are randomly chosen from $\left[0, q_{0}\right) \cap \mathbb{Z}$ (resp. $\left.\left(-2^{\rho}, 2^{\rho}\right) \cap \mathbb{Z}\right)$.

The secret-key is sk $=\{p\}$ and the public-key is pk $=\left\{x_{0}, x_{1}, \ldots, x_{\tau}, y, \boldsymbol{\sigma}, \boldsymbol{z}\right\}$.

SIDGHV. Encrypt(pk, $m \in\{0,1\}$ ). Choose a random subset $S \subset\{1, \ldots, \tau\}$ and output

$$
c \leftarrow\left[m \cdot y+\sum_{i \in S} x_{i}\right]_{x_{0}} .
$$

$\operatorname{SIDGHV} . \operatorname{Add}\left(\mathrm{pk}, c_{1}, c_{2}\right)$. Output $c \leftarrow c_{1}+c_{2} \bmod x_{0}$.

SIDGHV. Convert $(\mathrm{pk}, c)$. Output $c^{\prime} \leftarrow 2 \cdot\left\langle\boldsymbol{\sigma}, \operatorname{BitDecomp}_{\eta}(\boldsymbol{c})\right\rangle$ where $\boldsymbol{c}=(\lfloor c$. $\left.\left.z_{i}\right\rceil \bmod 2^{\eta}\right)_{1 \leqslant i \leqslant \Theta}$.

SIDGHV. Mult(pk, $\left.c_{1}, c_{2}\right)$. Output $\left.c^{\prime} \leftarrow \operatorname{SIDGHV.~Convert(pk,~} 2 \cdot c_{1} \cdot c_{2}\right) \bmod x_{0}$.

SIDGHV. Decrypt $($ sk,$c)$. Output $m \leftarrow((2 c) \bmod p) \bmod 2$.

Remark 1. This describes a leveled fully homomorphic encryption scheme, because the noise growth is only linear in the number of levels. The scheme can be bootstrapped to obtain a (pure) fully homomorphic encryption scheme, as in DGHV10, $\mathrm{CCK}^{+} 13$, 


\subsection{Constraints on the Parameters}

The parameters of the scheme must meet the following constraints (where $\lambda$ is the security parameter):

- $\rho=\Omega(\lambda)$ to avoid brute force attack on the noise [CN12, CNT12,

- $\eta \geqslant \rho+\mathcal{O}(L \log \lambda)$ where $L$ is the multiplicative depth of the circuit to be evaluated,

- $\gamma=\omega\left(\eta^{2} \cdot \log \lambda\right)$ in order to thwart lattice-based attacks (see [DGHV10] and CMNT11, CH12),

- $\Theta^{2}=\gamma \cdot \omega(\log \lambda)$ to avoid lattice attacks on the subset sum (see [CMNT11]),

- $\tau \geqslant \gamma+2 \lambda$ in order to apply the Leftover Hash Lemma (see Section 3.5).

To satisfy the above constraints one can take $\rho=2 \lambda, \eta=\tilde{\mathcal{O}}(L+\lambda), \gamma=$ $\tilde{\mathcal{O}}\left(L^{2} \lambda+\lambda^{2}\right), \Theta=\tilde{\mathcal{O}}(L \lambda)$ and $\tau=\gamma+2 \lambda$.

\subsection{Semantic Security}

We show that the semantic security of our scheme can be based on the following variant of the decisional problem introduced in KLYC13, called the DecisionalApproximate-GCD problem. Roughly speaking, it should be hard to distinguish integers from $\mathcal{D}_{p, q_{0}}^{\rho}$ from completely uniform integers modulo $x_{0}$, where:

$$
\mathcal{D}_{p, q_{0}}^{\rho}=\left\{q \cdot p^{2}+r: q \in \mathbb{Z} \cap\left[0, q_{0}\right), r \in \mathbb{Z} \cap\left(-2^{\rho}, 2^{\rho}\right)\right\} .
$$

Definition 2 ( $(\rho, \eta, \gamma)$-Decisional-Approximate-GCD). Let $p$ be a random odd integer of $\eta$ bits, $q_{0}$ an integer uniformly distributed in $\left[0,2^{\gamma} / p^{2}\right), r_{0}$ an integer uniformly distributed in $\left(-2^{\rho}, 2^{\rho}\right)$. Given $x_{0}=q_{0} \cdot p^{2}+r_{0}$, polynomially many samples from $\mathcal{D}_{p, q_{0}}^{\rho}$ and $y \leftarrow \mathcal{D}_{p, q_{0}}^{\rho}+(p-1) / 2$, determine $b \in\{0,1\}$ from $c=x+b \cdot r \bmod x_{0}$ where $x \leftarrow \mathcal{D}_{p, q_{0}}^{\rho}$ and $r \leftarrow\left[0, x_{0}\right) \cap \mathbb{Z}$.

The following theorem shows that our scheme is semantically secure under the Decisional-Approximate-GCD assumption; below we only consider a subset of our scheme without the procedure Convert, i.e. without the public parameters $\boldsymbol{z}$ and $\boldsymbol{\sigma}$. To prove the semantic security of the full scheme it suffices to include $\boldsymbol{z}$ and $\boldsymbol{\sigma}$ in the above decisional assumption 1

${ }^{1}$ Usually in FHE we first show the semantic security of a restricted scheme, and then a 'circular security' assumption is used to get the semantic security of the entire FHE; that is we assume that the encryption scheme remains secure even when the adversary is given encryptions of the individual bits of the private-key.

Here we first prove that the scheme is secure without the terms $\boldsymbol{z}$ and $\boldsymbol{\sigma}$. If the scheme is 'circular secure' (secure even with encryptions of the invariant switching, i.e. $\boldsymbol{z}$ and $\boldsymbol{\sigma}$ ) then it remains semantically secure. This circular security assumption can be avoided by using the classical modulus switching technique [CNT12] instead of our scale-invariance technique. 
Theorem 1. The above scale-invariant DGHV scheme without the parameters $\boldsymbol{z}, \boldsymbol{\sigma}$ is semantically secure under the $(\rho, \eta, \gamma)$-Decisional-Approximate-GCD assumption.

To prove the theorem, we use a preliminary Lemma from KLYC13 stating that the distribution of the public-key elements is indistinguishable from random elements in $\left[0, x_{0}\right)$ if the Decisional-Approximate-GCD problem is hard; the proof follows from a standard hybrid argument.

Lemma 2. For the parameters $(\rho, \eta, \gamma)$, let $\mathrm{pk}=\left(x_{0},\left\{x_{i}\right\}_{i}, y\right)$ and $\mathrm{sk}=p$ be chosen as in the KeyGen procedure. Define $\mathrm{pk}^{\prime}=\left(x_{0},\left\{x_{i}^{\prime}\right\}_{i}, y\right)$ for $x_{i}^{\prime}$ uniformly generated in $\left[0, x_{0}\right)$. Then $\mathrm{pk}$ and $\mathrm{pk}^{\prime}$ are indistinguishable under the DecisionalApproximate-GCD assumption.

Proof (of Theorem 11). Under the attack scenario the attacker first receives the public key, and an encryption of a random bit $b \in\{0,1\}$. The attacker outputs a guess $b^{\prime}$ and succeeds if $b^{\prime}=b$. We use a sequence of games and denote by $S_{i}$ the event that the attacker succeeds in $\mathbf{G a m e}_{i}$.

Game $_{0}$ : This is the attack scenario. We simulate the challenger by running KeyGen to obtain pk and sk.

Game $_{1}$ : We replace the $x_{i}$ 's in the public key by elements uniformly drawn in $\left[0, x_{0}\right)$. By Lemma 2, we have

$$
\left|\operatorname{Pr}\left[S_{1}\right]-\operatorname{Pr}\left[S_{0}\right]\right| \leqslant \tau \cdot \varepsilon_{\text {dagcd }} .
$$

Game $_{2}$ : By the Leftover Hash Lemma (Lemma 5 in Appendix $\mathrm{A}$ ), $\sum_{i \in S} x_{i} \bmod$ $x_{0}$ is $\varepsilon$-statistically indistinguishable from uniform modulo $x_{0}$, with $\varepsilon=2^{(\gamma-\tau) / 2}$. Therefore we can replace the challenge ciphertext by a uniform integer modulo $x_{0}$; this no longer gives any information on $b$ and therefore $\operatorname{Pr}\left[S_{2}\right]=1 / 2$. Moreover we have $\left|\operatorname{Pr}\left[S_{2}\right]-\operatorname{Pr}\left[S_{1}\right]\right| \leqslant \varepsilon$. This gap can be made negligible by satisfying the constraints on the parameters from Section 3.4, which concludes the proof.

Remark 2. We show in Section 6 that the (Error-Free) Decisional-ApproximateGCD problem is equivalent to the computational (Error-Free) ApproximateGCD problem. Thus our scheme is automatically based on the computational Approximate-GCD problem as in previous works on the DGHV schemes DGHV10, CMNT11, CNT12.

\section{Generalization to Batch Scale-Invariant DGHV Scheme}

We now describe a generalization of the previous scheme to the batch setting (as in RLWE-based schemes [BV11a, BV11b and integer schemes $\left.\mathrm{CCK}^{+} 13\right]$ ]. The goal is to pack $\ell$ plaintext bits $m_{0}, \ldots, m_{\ell-1}$ into a single ciphertext. Homomorphic addition and multiplication will then apply in parallel and component-wise on the $m_{i}$ 's. 
Our batch generalization is similar to $\left[\mathrm{CCK}^{+} 13\right.$. A ciphertext encrypting a vector $\boldsymbol{m}=\left(m_{0}, \ldots, m_{\ell-1}\right)$ has the form:

$$
c=\mathrm{CRT}_{q_{0}, p_{0}^{2}, \ldots, p_{\ell-1}^{2}}\left(q, \ldots, r_{i}+\left(2 r_{i}^{*}+m_{i}\right) \cdot \frac{p_{i}-1}{2}, \ldots\right)
$$

for a tuple of $\ell+1$ coprime integers $q_{0}, p_{0}, \ldots, p_{\ell-1}$, where we denote by $\operatorname{CRT}_{b_{i}}\left(a_{i}\right)$ the unique integer $u$ such that $0 \leqslant u<\prod_{i} b_{i}$ and $u \bmod b_{i}=a_{i}$ for all $i$. We call such ciphertext a batch Type-I ciphertext. Modulo each of the $p_{j}$ 's the ciphertext $c$ behaves as in the SIDGHV scheme in Section 3. Accordingly, the addition of two ciphertexts yields a new ciphertext that decrypts to the componentwise sum modulo 2 of the original plaintexts.

To homomorphically multiply two ciphertexts $c_{1}$ and $c_{2}$, as previously one computes $c_{3}=2 \cdot c_{1} \cdot c_{2}$ in $\mathbb{Z}$. As previously there exists small integers $r_{3, j}$ such that

$$
c_{3} \equiv r_{3, j}+m_{j} \cdot \frac{p_{j}^{2}-1}{2} \quad\left(\bmod p_{j}\right) \quad \text { for } j=0, \ldots, \ell-1,
$$

where each $m_{j}$ is the product of the corresponding plain text components of $c_{1}$ and $c_{2}$. We call $c_{3}$ a batch Type-II ciphertext. Modulo each of the $p_{j}$ 's, the ciphertext $c_{3}$ behaves as a Type-II ciphertext given by Equation (2); therefore the message bit $m_{j}$ is the MSB of $\left[c \bmod p_{j}^{2}\right]$ for all $j$. As in Section 3 , there exists an efficient conversion procedure Convert to convert any Type-II ciphertext to a new Type-I ciphertext. As shown below the procedure Convert is actually the same as in Section 3, with adapted public parameters.

Namely let $\boldsymbol{z}$ be a vector of $\Theta$ rational numbers in $\left[0,2^{\eta}\right)$ with $\kappa$ bits of precision after the binary point (where $|c|<2^{\kappa}$ ), and let $\left(s_{j}\right)$ be a set of $\ell$ vectors of $\Theta$ bits such that, for all $j=0, \ldots, \ell-1$,

$$
\frac{2^{\eta}}{p_{j}^{2}}=\left\langle\boldsymbol{s}_{j}, \boldsymbol{z}\right\rangle+\varepsilon_{j} \bmod 2^{\eta}
$$

where $\left|\varepsilon_{j}\right| \leqslant 2^{-\kappa}$. Let $\boldsymbol{s}_{j}^{\prime}=\operatorname{PowersofT}_{w_{\eta}}\left(\boldsymbol{s}_{j}\right) \in \mathbb{Z}^{\eta \Theta}$. Define $\boldsymbol{\sigma}=\left(\sigma_{1}, \ldots, \sigma_{\eta \Theta}\right)$ so that, for all $1 \leqslant i \leqslant \eta \Theta$ :

$$
\sigma_{i}=\mathrm{CRT}_{q_{0}, p_{0}^{2}, \ldots, p_{\ell-1}^{2}}\left(q_{i}, r_{0, i}+\left\lfloor s_{0, i}^{\prime} \cdot \frac{p_{0}}{2^{\eta+1}}\right\rceil, \ldots, r_{\ell-1, i}+\left\lfloor s_{\ell-1, i}^{\prime} \cdot \frac{p_{\ell-1}}{2^{\eta+1}}\right\rceil\right)
$$

is an encryption of $\left(s_{j, i}^{\prime}\right)_{1 \leqslant j \leqslant \ell}$. For Convert we use the same algorithm as in Section 3

Convert $(\boldsymbol{z}, \boldsymbol{\sigma}, c)$. First compute $\boldsymbol{c}=\left(\left\lfloor c \cdot z_{i}\right\rceil \bmod 2^{\eta}\right)_{1 \leqslant i \leqslant \Theta}$ and then its decomposition $\boldsymbol{c}^{\prime}=$ BitDecomp $_{\eta}(\boldsymbol{c})$. Finally, output

$$
c^{\prime} \leftarrow 2\left\langle\boldsymbol{\sigma}, \boldsymbol{c}^{\prime}\right\rangle \bmod x_{0}
$$

The proof of the following lemma follows directly from the proof of Lemma 1 applied modulo each of the $p_{j}$ 's. 
Lemma 3. The procedure Convert above converts a Type-II ciphertext with noise size $\rho^{\prime}$ into a Type-I ciphertext with noise $\left(\rho^{\prime}-\eta+5, \log _{2} \Theta\right)$, for $\rho^{\prime}-\eta \geqslant$ $\rho+\log _{2}(\eta \Theta)$.

In the full version of this paper CLT14 we provide a full description of the resulting batch leveled fully homomorphic scheme. We also show that the batch scheme is semantically secure under a variant of the previous Decisional-Approximate-GCD assumption with error-free $x_{0}$.

\section{Practical Implementation}

In this section, we provide concrete parameters and timings for a homomorphic evaluation of AES with our batch scale-invariant DGHV scheme. For homomorphic AES evaluations we compare our timings with the RLWE-based leveledFHE scheme in GHS12b and with the batch (bootstrapping-based) DGHV scheme in $\mathrm{CCK}^{+} 13$, CLT13. We use the following existing optimizations:

1. Subset-sum: as in CMNT11 we use $\beta$-bit integers $b_{i}$ instead of bits in the subset sum, to reduce the value of $\tau$. Namely the condition becomes $\beta \cdot \tau \geqslant$ $\gamma+2 \lambda$.

2. Public-key compression: the technique in CNT12, CLT13, enables to compress the ciphertexts in the public-key from $\gamma$ to roughly $\ell \cdot \eta$ bits.

3. Ciphertext expand [CNT12]: the technique consists in generating the $z_{i}$ 's with a special structure instead of pseudo-random. Let $\delta$ be a parameter to be specified later. One generates a random $z$ with $\kappa+\delta \cdot \Theta \cdot \eta$ bits of precision after the binary point, and one defines the $z_{i}$ 's for $\ell+1 \leqslant i \leqslant \Theta$ as

$$
z_{i}=\left[z \cdot 2^{i \cdot \delta \cdot \eta}\right]_{2^{\eta}},
$$

keeping only $\kappa$ bits of precision after the binary point for each $z_{i}$ as previously. We fix $z_{1}, \ldots, z_{\ell}$ so that the previous equalities hold. Then the ciphertext expansion can be computed as follows, for all $\ell+1 \leqslant i \leqslant \Theta$ :

$$
c_{i}=\left\lfloor c \cdot z_{i}\right\rceil \bmod 2^{\eta}=\left\lfloor c \cdot z \cdot 2^{i \cdot \delta \cdot \eta}\right\rceil \bmod 2^{\eta} .
$$

Therefore computing all the $z_{i}$ 's (except the first $\ell$ ) is now essentially a single multiplication $c \cdot z$. A lattice attack against this optimization is described in CNT12; the authors show that the attack is thwarted by selecting $\delta$ such that $\delta \cdot \Theta \cdot \eta \geqslant 3 \gamma$.

\subsection{Optimization of Scalar Product}

We describe an additional optimization for computing the scalar product $c^{\prime}=$ $2\left\langle\boldsymbol{\sigma}, \boldsymbol{c}^{\prime}\right\rangle$ computed in Convert, similar to the ciphertext expand optimization above. The vectors $\boldsymbol{\sigma}$ and $\boldsymbol{c}^{\prime}$ have $\eta \Theta$ elements. We first divide the vectors $\boldsymbol{\sigma}$ and $\boldsymbol{c}^{\prime}$ into subvectors of $\Theta$ elements, and we compute the scalar products of the 
subvectors separately. In the following for simplicity we keep the same notations and now assume that $\boldsymbol{\sigma}$ and $\boldsymbol{c}^{\prime}$ have $\Theta$ elements each.

We generate the vector $\boldsymbol{\sigma} \in \mathbb{Z}^{\Theta}$ such that:

$$
\sigma_{i}=\left\lfloor\sigma \cdot 2^{i \cdot \delta \cdot \eta}\right\rceil+v_{i}
$$

for small public corrections $\left|v_{i}\right| \leqslant 2^{\eta \cdot \ell}$ for all $1 \leqslant i \leqslant \Theta$, where the large public random $\sigma$ has $\delta \eta \Theta$ bits of precision after the binary point, and $\gamma+\delta \eta \Theta$ bits in total. Then

$$
\begin{aligned}
c^{\prime} & =2\left\langle\boldsymbol{\sigma}, \boldsymbol{c}^{\prime}\right\rangle=2 \sum_{i=1}^{n}\left\lfloor\sigma \cdot 2^{i \cdot \delta \cdot \eta}\right\rceil \cdot c_{i}^{\prime}+2\left\langle\boldsymbol{v}, \boldsymbol{c}^{\prime}\right\rangle=2 \sum_{i=1}^{n}\left(\sigma \cdot 2^{i \delta \eta}+u_{i}\right) c_{i}^{\prime}+2\left\langle\boldsymbol{v}, \boldsymbol{c}^{\prime}\right\rangle \\
& =2 \sigma \cdot\left(\sum_{i=1}^{n} c_{i}^{\prime} \cdot 2^{i \delta \eta}\right)+2\left\langle\boldsymbol{v}, \boldsymbol{c}^{\prime}\right\rangle+u=\left\lfloor 2 \sigma \cdot\left(\sum_{i=1}^{n} c_{i}^{\prime} \cdot 2^{i \delta \eta}\right)\right\rceil+2\left\langle\boldsymbol{v}, \boldsymbol{c}^{\prime}\right\rangle+u^{\prime},
\end{aligned}
$$

where $\left|u_{i}\right| \leqslant 1 / 2,|u| \leqslant \Theta$, and $u^{\prime} \in \mathbb{Z}$ is such that $\left|u^{\prime}\right| \leqslant \Theta+1$. Then the scalar product becomes essentially one multiplication and another scalar product but with much smaller entries $v_{i}$ 's instead of $\sigma_{i}$ 's.

Therefore with vectors $\boldsymbol{\sigma}$ and $\boldsymbol{c}^{\prime}$ with $\eta \Theta$ elements each instead of $\Theta$, the scalar product $2\left\langle\boldsymbol{\sigma}, \boldsymbol{c}^{\prime}\right\rangle$ becomes essentially $\eta$ multiplications and another scalar product but with much smaller entries $v_{i}$ 's instead of $\sigma_{i}$ 's. Note that the size of $c^{\prime}$ is now $\gamma+\Theta \delta \eta$ bits instead of $\gamma$; therefore one must increase $\kappa$ by twice the same additive factor (to support multiplications of two such converted ciphertexts).

Finally we use the following straightforward optimization: instead of using BitDecomp and PowersofTwo with bits, we use words of size $\omega$ bits instead. This decreases the size of the vector $\boldsymbol{\sigma}$ by a factor $\omega$, at the cost of increasing the resulting noise by roughly $\omega$ bits. In particular the scalar product $2\left\langle\boldsymbol{\sigma}, \boldsymbol{c}^{\prime}\right\rangle$ then requires essentially $\lceil\eta / \omega\rceil$ multiplications and another scalar product but with smaller entries $v_{i}$ 's instead of $\sigma_{i}$ 's. In our code we used $\omega=64$.

\subsection{Concrete Parameters and AES Evaluation}

In Table 1 we derive concrete parameters as in $\mathrm{CNT12}_{\mathrm{CCK}}^{+}$13], taking into account the known attacks on the Approximate-GCD problem (see [DGHV10] and [CMNT11, CNT12, CN12, CH12]).

AES evaluation has become a standard evaluation circuit for fully homomorphic encryption GHS12b, $\mathrm{CCK}^{+} 13$. The main difference between GHS12b and $\left[\mathrm{CCK}^{+} 13\right]$ (apart from the underlying FHE scheme) is that bootstrapping was used in the later while in the former the parameters could be made large enough so that no bootstrapping was required to evaluate the full-fledged AES circuit (thanks to the linear growth of the noise). In our scheme we also chose large enough parameters so that the entire AES evaluation could be performed without bootstrapping.

In practice we have evaluated the AES circuit using the state-wise bitslicing variant described in [CLT13] and we obtained the results in Table 1] In this variant, the state is represented as an array of 128 ciphertexts, each ciphertext 
Table 1. Benchmarking of a $\mathrm{C}++$ implementation of our scale-invariant batch DGHV scheme with a compressed public key on an Intel Xeon E5-2690 at $2.9 \mathrm{GHz}$ on the state-wise AES implementation, using GMP Gt13.

\begin{tabular}{|l|c|c||c|c|c|c|c||c|c|c|c|c|}
\hline Instance & $\lambda$ & $\ell$ & $\rho$ & $\eta$ & $\gamma \times 10^{-6}$ & $\tau, \Theta$ & pk size & KeyGen & Encrypt & Decrypt & Mult & Convert \\
\hline \hline Toy & 42 & 9 & 42 & 971 & 0.27 & 135 & $3.2 \mathrm{MB}$ & $0.5 \mathrm{~s}$ & $0.0 \mathrm{~s}$ & $0.0 \mathrm{~s}$ & $0.0 \mathrm{~s}$ & $0.1 \mathrm{~s}$ \\
\hline Small & 52 & 35 & 52 & 976 & 1.1 & 525 & $45 \mathrm{MB}$ & $11 \mathrm{~s}$ & $0.2 \mathrm{~s}$ & $0.0 \mathrm{~s}$ & $0.0 \mathrm{~s}$ & $0.3 \mathrm{~s}$ \\
\hline Medium & 62 & 140 & 62 & 981 & 4.2 & 2100 & $704 \mathrm{MB}$ & $5 \mathrm{~min}$ & $3 \mathrm{~s}$ & $0.2 \mathrm{~s}$ & $0.0 \mathrm{~s}$ & $2.8 \mathrm{~s}$ \\
\hline Large & 72 & 569 & 72 & 986 & 15.8 & 8535 & $11 \mathrm{~GB}$ & $2 \mathrm{~h} 50 \mathrm{~min}$ & $45 \mathrm{~s}$ & $3.3 \mathrm{~s}$ & $0.1 \mathrm{~s}$ & $33 \mathrm{~s}$ \\
\hline Extra & 80 & 1875 & 86 & 993 & 35.9 & 28125 & $100 \mathrm{~GB}$ & $213 \mathrm{~h}$ & $5 \mathrm{~min}$ & $24 \mathrm{~s}$ & $0.3 \mathrm{~s}$ & $277 \mathrm{~s}$ \\
\hline
\end{tabular}

\begin{tabular}{|l|c|c|c|c|c|c|c|c|}
\hline Instance & $\lambda$ & $\begin{array}{c}\ell=\text { \# of enc. } \\
\text { in parallel }\end{array}$ & AddRoundKey & SubBytes & ShiftRows & MixColumns & $\begin{array}{c}\text { Total } \\
\text { Time }\end{array}$ & $\begin{array}{c}\text { Time/AES } \\
\text { block }\end{array}$ \\
\hline Toy & 42 & 9 & $0.0 \mathrm{~s}$ & $1.5 \mathrm{~s}$ & $0.0 \mathrm{~s}$ & $0.0 \mathrm{~s}$ & $15.1 \mathrm{~s}$ & $\mathbf{1 . 7 s}$ \\
\hline Small & 52 & 35 & $0.1 \mathrm{~s}$ & $9.9 \mathrm{~s}$ & $0.0 \mathrm{~s}$ & $0.0 \mathrm{~s}$ & $1 \mathrm{~min} 40 \mathrm{~s}$ & $\mathbf{2 . 9 s}$ \\
\hline Medium & 62 & 140 & $0.3 \mathrm{~s}$ & $80.5 \mathrm{~s}$ & $0.0 \mathrm{~s}$ & $0.1 \mathrm{~s}$ & $13 \mathrm{~min} 29 \mathrm{~s}$ & $\mathbf{5 . 8 s}$ \\
\hline Large & 72 & 569 & $2.1 \mathrm{~s}$ & $21 \mathrm{~min}$ & $0.0 \mathrm{~s}$ & $0.6 \mathrm{~s}$ & $3 \mathrm{~h} 35 \mathrm{~min}$ & $\mathbf{2 3 s}$ \\
\hline Extra & 80 & 1875 & $6.9 \mathrm{~s}$ & $10 \mathrm{~h} 9 \mathrm{~min}$ & $0.1 \mathrm{~s}$ & $1.6 \mathrm{~s}$ & $102 \mathrm{~h}$ & $\mathbf{1 9 5}$ \\
\hline
\end{tabular}

representing one bit of the state of $\ell$ different AES blocks encrypted in parallel. In $\mathrm{CCK}^{+} 13$, CLT13, the authors obtained a time per AES block of 12 min $46 \mathrm{~s}$ on a 4 -core machine at $3.4 \mathrm{GHz}$ whereas we obtained $23 \mathrm{~s}$ on a 16 -core machine at $2.9 \mathrm{GHz}$ for the same security level (72 bits of security); which is one order of magnitude faster. For 80 bits of security, timings are competitive with GHS12b] (3 $\mathrm{min}$ vs. $5 \mathrm{~min})$.

\section{Equivalence between the (Error-Free) Decisional and Computational Approximate-GCD Problems}

In this section, we show the equivalence between the (error-free) decisional and computational Approximate-GCD problems. As a consequence, it follows directly that the additional noises in the fully homomorphic encryption schemes over the integers DGHV10, CMNT11, CNT12, CLT13 can be removed (as in $\mathrm{CCK}^{+} 13$, Section 3]), simplifying both the schemes and the security proofs. In the following for simplicity we only consider integers $r \in\left[0,2^{\rho}\right)$ instead of $\left(-2^{\rho}, 2^{\rho}\right)$. One can always go from one distribution to another by an appropriate centering. Therefore, for a $\eta$-bit integer $p$ and $q_{0} \in\left[0,2^{\gamma} / p\right)$, we consider the following distribution over $\gamma$-bit integers:

$$
\mathcal{D}_{\rho}\left(p, q_{0}\right)=\left\{\text { Choose } q \leftarrow\left[0, q_{0}\right), r \leftarrow \mathbb{Z} \cap\left[0,2^{\rho}\right): \text { Output } y=q \cdot p+r\right\} \text {. }
$$

Let us recall the definition of the computational and decisional Error-Free Approximate-GCD problems.

Definition 3 (Error-Free (Computational) Approximate-GCD). The ( $\rho$, $\eta, \gamma)$-error-free Approximate-GCD problem is: For a random $\eta$-bit prime $p$, given a $\gamma$-bit $2^{\lambda^{2}}$-rough integer $x_{0}=q_{0} \cdot p$ where $q_{0}$ is a random integer in $\left[0,2^{\gamma} / p\right)$, and polynomially many samples from $\mathcal{D}_{\rho}\left(p, q_{0}\right)$, output $p$. 


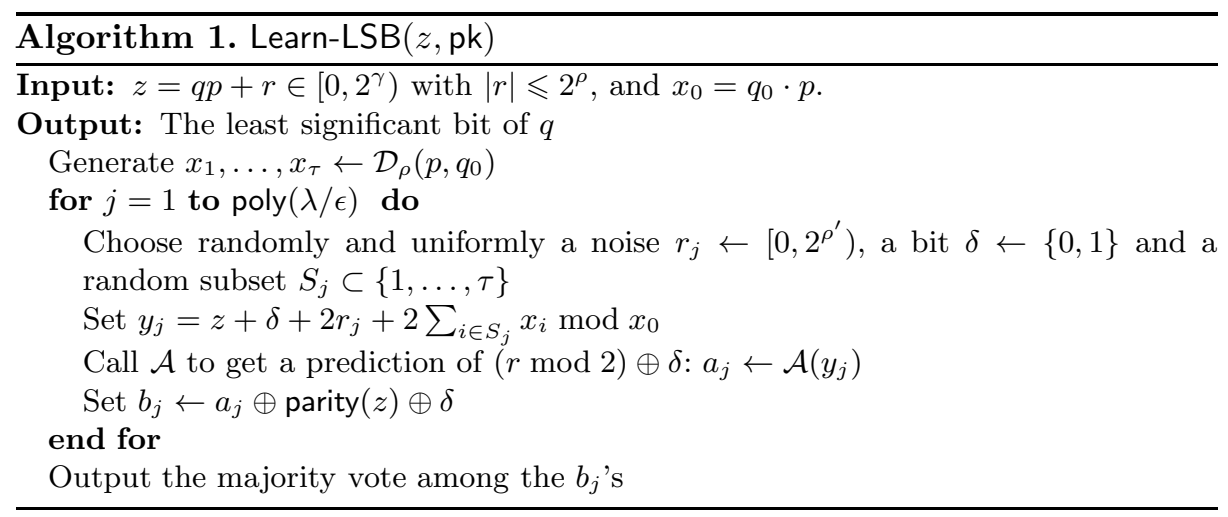

Definition 4 (Error-Free Decisional Approximate-GCD). The $(\rho, \eta, \gamma)$ error-free Decisional-Approximate-GCD problem is: For a random $\eta$-bit prime $p$, given a $\gamma$-bit $2^{\lambda^{2}}$-rough integer $x_{0}=q_{0} \cdot p$ and polynomially many samples from $\mathcal{D}_{\rho}\left(p, q_{0}\right)$, determine $b \in\{0,1\}$ from $z=x+r \cdot b \bmod x_{0}$ where $x \leftarrow \mathcal{D}_{\rho}\left(p, q_{0}\right)$ and $r \leftarrow \mathbb{Z} \cap\left[0, x_{0}\right)$.

We also consider the following decisional problem.

Definition 5 (Error-Free LSB Approximate-GCD Problem). The ( $\rho, \eta$, $\gamma)$-error-free LSB Approximate-GCD problem is: For a random $\eta$-bit prime $p$, given a $\gamma$-bit $2^{\lambda^{2}}$-rough integer $x_{0}=q_{0} \cdot p$ and polynomially many samples from $\mathcal{D}_{\rho}\left(p, q_{0}\right)$, determine $b \in\{0,1\}$ from $z=q \cdot p+2 r+b \cdot c$ where $q \leftarrow\left[0, q_{0}\right)$, $r \leftarrow \mathbb{Z} \cap\left[0,2^{\rho-1}\right)$ and $c \leftarrow\{0,1\}$.

One can show that the problems from Definitions 3 and 5 are equivalent. Indeed, we can construct a high-accuracy LSB predictor subroutine (cf. Algorithm 1 below) using an adversary $\mathcal{A}$ having a non-negligible advantage $\epsilon$ against the $\left(\rho^{\prime}, \eta, \gamma\right)$-Error-Free LSB Approximate-GCD problem (with $\rho^{\prime}>$ $\left.\log _{2}(\tau+1)+\rho+\lambda\right)$, and by using it in Step 2 of the security proof of [DGHV10], we automatically get the equivalence.

Let us show that Definitions 4 and 5 are equivalent. We consider the sequence of distributions for $\rho \leqslant i \leqslant \eta+\lambda$ :

$$
\mathcal{D}_{\rho}^{\prime}\left(p, q_{0}, i\right)=\left\{\begin{array}{c}
\text { Choose } q \leftarrow\left[0, q_{0}\right), r \leftarrow \mathbb{Z} \cap\left[0,2^{i}\right): \\
\text { Output } y=q \cdot p+2^{\lambda+\eta-i} \cdot r \bmod x_{0}
\end{array}\right\} .
$$

Note that in the distribution $\mathcal{D}_{\rho}^{\prime}\left(p, q_{0}, i\right)$ above the size of the random $r$ is $i$-bit instead of $\rho$-bit. For $i=\rho$, the distribution of $y$ is the same as the distribution $\mathcal{D}_{\rho}\left(p, q_{0}\right)$, up to a factor $2^{\lambda+\eta-\rho}$ modulo $x_{0}$. One can show that for $i=\eta+\lambda$, the distribution $\mathcal{D}_{\rho}^{\prime}\left(p, q_{0}, i\right)$ is $2^{-\lambda}$-statistically close to uniform modulo $x_{0}$. Therefore by a standard hybrid argument, if a distinguisher solves the

${ }^{2}$ The additional noise is use to drawn the noise due to the public key elements and $z$. 
Error-Free Decisional-Approximate-GCD problem with some non-negligible advantage, then he must be able to distinguish between two successive distributions $\mathcal{D}_{\rho}^{\prime}\left(p, q_{0}, i\right)$ and $\mathcal{D}_{\rho}^{\prime}\left(p, q_{0}, i+1\right)$ for some $i$.

Let us consider the challenge from the Error-Free LSB Approximate-GCD problem:

$$
z=q \cdot p+2 r+b \cdot c
$$

where $r \leftarrow \mathbb{Z} \cap\left[0,2^{\rho-1}\right)$ and $c \leftarrow\{0,1\}$. We let:

$$
y=2^{\lambda+\eta-i-1} \cdot\left(2^{\rho} \cdot u+z\right) \bmod x_{0}
$$

where $u \leftarrow \mathbb{Z} \cap\left[0,2^{i+1-\rho}\right)$. This gives:

$$
\begin{aligned}
y & =q^{\prime} \cdot p+2^{\lambda+\eta-i-1} \cdot\left(2^{\rho} \cdot u+2 r+b \cdot c\right) \bmod x_{0} \\
& =q^{\prime} \cdot p+2^{\lambda+\eta-i-1} \cdot\left(2 r^{\prime}+b \cdot c\right)
\end{aligned}
$$

for some $q^{\prime} \in \mathbb{Z}$, where $r^{\prime} \leftarrow \mathbb{Z} \cap\left[0,2^{i}\right)$.

If $b=0$ then we get $y=q^{\prime} \cdot p+2^{\lambda+\eta-i} \cdot r^{\prime}$ which corresponds to the distribution $\mathcal{D}_{\rho}^{\prime}\left(p, q_{0}, i\right)$. If $b=1$ then we get $y=q^{\prime} \cdot p+2^{\lambda+\eta-i-1} \cdot r^{\prime \prime}$ where $r^{\prime \prime} \leftarrow \mathbb{Z} \cap\left[0,2^{i+1}\right)$, which corresponds to the distribution $\mathcal{D}_{\rho}^{\prime}\left(p, q_{0}, i+1\right)$. Therefore we can use the previous distinguisher to solve the Error-Free LSB Approximate-GCD problem.

\section{References}

[BGV12] Brakerski, Z., Gentry, C., Vaikuntanathan, V.: (Leveled) fully homomorphic encryption without bootstrapping. In: Goldwasser, S. (ed.) ITCS 2012, pp. 309-325. ACM (2012)

[Bra12] Brakerski, Z.: Fully homomorphic encryption without modulus switching from classical GapSVP. In: Safavi-Naini, R., Canetti, R. (eds.) CRYPTO 2012. LNCS, vol. 7417, pp. 868-886. Springer, Heidelberg (2012)

[BV11a] Brakerski, Z., Vaikuntanathan, V.: Efficient fully homomorphic encryption from (standard) LWE. In: FOCS 2011, pp. 97-106. IEEE Computer Society (2011)

[BV11b] Brakerski, Z., Vaikuntanathan, V.: Fully homomorphic encryption from Ring-LWE and security for key dependent messages. In: Rogaway, P. (ed.) CRYPTO 2011. LNCS, vol. 6841, pp. 505-524. Springer, Heidelberg (2011)

$\left[\mathrm{CCK}^{+} 13\right]$ Cheon, J.H., Coron, J.-S., Kim, J., Lee, M.S., Lepoint, T., Tibouchi, M., Yun, A.: Batch fully homomorphic encryption over the integers. In: Johansson, T., Nguyen, P.Q. (eds.) EUROCRYPT 2013. LNCS, vol. 7881, pp. 315-335. Springer, Heidelberg (2013)

[CH12] Cohn, H., Heninger, N.: Approximate common divisors via lattices. In: ANTS X (2012)

[CLT13] Coron, J.-S., Lepoint, T., Tibouchi, M.: Batch fully homomorphic encryption over the integers. Cryptology ePrint Archive, Report 2013/036 (2013), http://eprint.iacr.org/

[CLT14] Coron, J.-S., Lepoint, T., Tibouchi, M.: Scale-invariant fully homomorphic encryption over the integers. Full version of this paper. Cryptology ePrint Archive, Report 2014/032 (2014), http://eprint.iacr.org/ 
[CMNT11] Coron, J.-S., Mandal, A., Naccache, D., Tibouchi, M.: Fully homomorphic encryption over the integers with shorter public keys. In: Rogaway, P. (ed.) CRYPTO 2011. LNCS, vol. 6841, pp. 487-504. Springer, Heidelberg (2011)

[CN12] Chen, Y., Nguyen, P.Q.: Faster algorithms for approximate common divisors: Breaking fully-homomorphic-encryption challenges over the integers. In: Pointcheval, D., Johansson, T. (eds.) EUROCRYPT 2012. LNCS, vol. 7237, pp. 502-519. Springer, Heidelberg (2012)

[CNT12] Coron, J.-S., Naccache, D., Tibouchi, M.: Public key compression and modulus switching for fully homomorphic encryption over the integers. In: Pointcheval, D., Johansson, T. (eds.) EUROCRYPT 2012. LNCS, vol. 7237, pp. 446-464. Springer, Heidelberg (2012)

[DGHV10] van Dijk, M., Gentry, C., Halevi, S., Vaikuntanathan, V.: Fully homomorphic encryption over the integers. In: Gilbert, H. (ed.) EUROCRYPT 2010. LNCS, vol. 6110, pp. 24-43. Springer, Heidelberg (2010)

[Gen09] Gentry, C.: Fully homomorphic encryption using ideal lattices. In: Mitzenmacher, M. (ed.) STOC, pp. 169-178. ACM (2009)

[GH11] Gentry, C., Halevi, S.: Implementing Gentry's fully-homomorphic encryption scheme. In: Paterson, K.G. (ed.) EUROCRYPT 2011. LNCS, vol. 6632, pp. 129-148. Springer, Heidelberg (2011)

[GHS12a] Gentry, C., Halevi, S., Smart, N.P.: Fully homomorphic encryption with polylog overhead. In: Pointcheval, D., Johansson, T. (eds.) EUROCRYPT 2012. LNCS, vol. 7237, pp. 465-482. Springer, Heidelberg (2012)

[GHS12b] Gentry, C., Halevi, S., Smart, N.P.: Homomorphic evaluation of the AES circuit. In: Safavi-Naini, R., Canetti, R. (eds.) CRYPTO 2012. LNCS, vol. 7417, pp. 850-867. Springer, Heidelberg (2012)

[Gt13] Torbjörn Granlund and the GMP development team. GNU MP: The GNU Multiple Precision Arithmetic Library, 5.1.3 edition (2013), http://gmplib.org/

[HILL99] Håstad, J., Impagliazzo, R., Levin, L.A., Luby, M.: A pseudorandom generator from any one-way function. SIAM Journal on Computing 28, 12-24 (1999)

[KLYC13] Kim, J., Lee, M.S., Yun, A., Cheon, J.H.: CRT-based fully homomorphic encryption over the integers. Cryptology ePrint Archive, Report 2013/057 (2013), http://eprint.iacr.org/

[Reg05] Regev, O.: On lattices, learning with errors, random linear codes, and cryptography. In: Gabow, H.N., Fagin, R. (eds.) STOC 2005, pp. 84-93. ACM (2005)

[SV10] Smart, N.P., Vercauteren, F.: Fully homomorphic encryption with relatively small key and ciphertext sizes. In: Nguyen, P.Q., Pointcheval, D. (eds.) PKC 2010. LNCS, vol. 6056, pp. 420-443. Springer, Heidelberg (2010)

[SV11] Smart, N.P., Vercauteren, F.: Fully homomorphic SIMD operations. IACR Cryptology ePrint Archive, 2011:133 (2011)

\section{A Leftover Hash Lemma}

We recall the classical Leftover Hash Lemma (LHL), following [DGHV10]. A family $\mathcal{H}$ of hash functions from $X$ to $Y$, both finite sets, is said to be pairwiseindependent if for all distinct $x, x^{\prime} \in X, \operatorname{Pr}_{h \leftarrow H}\left[h(x)=h\left(x^{\prime}\right)\right]=1 /|Y|$. A distribution $D$ is $\varepsilon$-uniform if its statistical distance from the uniform distribution 
is at most $\varepsilon$, where the statistical distance $\Delta\left(D_{1}, D_{2}\right)$ between two distributions $D_{1}, D_{2}$ over a finite domain $X$ is given by $\Delta\left(D_{1}, D_{2}\right)=\frac{1}{2} \sum_{x \in X}\left|D_{1}(x)-D_{2}(x)\right|$.

Lemma 4 (Leftover Hash Lemma [HILL99]). Let $\mathcal{H}$ be a family of pairwise hash functions from $X$ to $Y$. Suppose that $h \leftarrow \mathcal{H}$ and $x \leftarrow X$ are chosen uniformly and independently. Then, $(h, h(x))$ is $\frac{1}{2} \sqrt{|Y| /|X|}$-uniform over $\mathcal{H} \times X$.

From the LHL one can deduce the following Lemma for finite sums modulo an integer $M$, as proved in DGHV10]:

Lemma 5. Set $x_{1}, \ldots, x_{m} \leftarrow \mathbb{Z}_{M}$ uniformly and independently, set $s_{1}, \ldots, s_{m} \leftarrow$ $\{0,1\}$, and set $y=\sum_{i=1}^{m} s_{i} \cdot x_{i} \bmod M$. Then $\left(x_{1}, \ldots, x_{m}, y\right)$ is $1 / 2 \sqrt{M / 2^{m}}$. uniform over $\mathbb{Z}_{M}^{m+1}$.

Proof. We consider the following hash function family $\mathcal{H}$ from $\{0,1\}^{m}$ to $\mathbb{Z}_{M}$. Each member $h \in \mathcal{H}$ is parameterized by the elements $\left(x_{1}, \ldots, x_{m}\right) \in \mathbb{Z}_{M}^{m}$. Given $s \in\{0,1\}^{m}$, we define $h(s)=\sum_{i=1}^{m} s_{i} \cdot x_{i} \in \mathbb{Z}_{M}$. The hash function family is clearly pairwise independent. Therefore by Lemma 4 , $(h, h(x))$ is $1 / 2 \sqrt{M / 2^{m}}$ uniform over $\mathbb{Z}_{M}^{m+1}$. 